\title{
Observing coseismic gravity change from the Japan Tohoku-Oki 2011 earthquake with GOCE gravity gradiometry
}

\author{
Martin J. Fuchs, ${ }^{1}$ Johannes Bouman, ${ }^{1}$ Taco Broerse, ${ }^{2,3}$ Pieter Visser, ${ }^{3}$ and Bert Vermeersen ${ }^{3,4}$ \\ Received 5 January 2013; revised 18 September 2013; accepted 19 September 2013.
}

[1] The Japan Tohoku-Oki earthquake $(9.0 \mathrm{Mw})$ of 11 March 2011 has left signatures in the Earth's gravity field that are detectable by data of the Gravity field Recovery and Climate Experiment (GRACE) mission. Because the European Space Agency's (ESA) satellite gravity mission Gravity field and steady-state Ocean Circulation Explorer (GOCE) - launched in 2009 - aims at high spatial resolution, its measurements could complement the GRACE information on coseismic gravity changes, although time-variable gravity was not foreseen as goal of the GOCE mission. We modeled the coseismic earthquake geoid signal and converted this signal to vertical gravity gradients at GOCE satellite altitude. We combined the single gradient observations in a novel way reducing the noise level, required to detect the coseismic gravity change, subtracted a global gravity model, and applied tailored outlier detection to the resulting gradient residuals. Furthermore, the measured gradients were along-track filtered using different gradient bandwidths where in the space domain Gaussian smoothing has been applied. One-year periods before and after earthquake occurrence have been compared with the modeled gradients. The comparison reveals that the earthquake signal is well above the accuracy of the vertical gravity gradients at orbital height. Moreover, the obtained signal from GOCE shows a 1.3 times higher amplitude compared with the modeled signal. Besides the statistical significance of the obtained signal, it has a high spatial correlation of $\sim 0.7$ with the forward modeled signal. We conclude therefore that the coseismic gravity change of the Japan Tohoku-Oki earthquake left a statistically significant signal in the GOCE measured gravity gradients.

Citation: Fuchs, M. J., J. Bouman, T. Broerse, P. Visser, and B. Vermeersen (2013), Observing coseismic gravity change from the Japan Tohoku-Oki 2011 earthquake with GOCE gravity gradiometry, J. Geophys. Res. Solid Earth, 118, doi: $10.1002 /$ jgrb.50381.

\section{Introduction}

[2] The objective of the European Space Agency's (ESA) GOCE (Gravity field and steady-state Ocean Circulation Explorer) mission is to determine the Earth's time-averaged gravity field (considered as static) with an accuracy of $1-2 \mathrm{~cm}$ in terms of geoid heights and $1 \mathrm{mGal}$ in terms of gravity anomalies down to $100 \mathrm{~km}$ resolution [European Space Agency, 1999]. To achieve this demanding goal, GOCE is currently the lowest flying satellite at a nominal orbit height of about $260 \mathrm{~km}$. The key instrument onboard GOCE is a gravity gradiometer from which GOCE's main observations, the gravity gradients or the second spatial derivatives of the Earth's gravitational potential, are being derived.

\footnotetext{
${ }^{1}$ Deutsches Geodätisches Forschungsinstitut, Munich, Germany.

${ }^{2}$ Department of Geosciences and Remote Sensing, Delft University of Technology, Delft, Netherlands.

${ }^{3}$ Faculty of Aerospace Engineering, Delft University of Technology, Delft, Netherlands.

${ }^{4}$ NIOZ, Texel, The Netherlands.

Corresponding author: M. Fuchs, Deutsches Geodätisches Forschungsinstitut, Alfons-Goppel-Str. 11, Munich, 80539, Germany. (fuchs@dgfi.badw.de)

(C)2013. American Geophysical Union. All Rights Reserved.

2169-9313/13/10.1002/jgrb.50381
}

[3] GOCE never intended to derive the global time-variable gravity field since a spaceborne gradiometer is especially sensitive to signals at spatial resolutions of $1500 \mathrm{~km}$ or smaller, whereas temporal gravity signals, e.g., due to hydrology, tides, etc., commonly have most power at long wavelengths below the gradiometer sensitivity [Abrikosov et al., 2006; Bouman et al., 2009, 2011; Han et al., 2006]. In contrast, megathrust earthquakes cause large deformations and related sudden geoid changes, which typically have a spatial signature of $500 \mathrm{~km}$ or less and are modeled to be at centimeter level [e.g., Broerse et al., 2011b; Zhou et al., 2012].

[4] The mass displacement of both the Maule 2010 and Tohoku-Oki 2011 events caused changes in the Earth's gravitational field that have been detected by the satellite gravity mission GRACE (Gravity field Recovery and Climate Experiment), [see Heki and Matsuo, 2010; Han et al., 2011; Matsuo and Heki, 2011; Wang et al., 2012; Zhou et al., 2012]. An assessment of the feasibility to detect these earthquakes in the GOCE gravity gradients showed that this primarily depends on the amount of accumulated data [Fuchs et al., 2012]. For the Japan Tohoku-Oki earthquake, approximately 1 year of reprocessed GOCE data is available before and after the earthquake. For the Chile Maule earthquake, on the other hand, only 3 months of data before the earthquake are available. Moreover, the coseismic gravity 
signal is approximately half for the Chile Maule earthquake compared with the Japan Tohoku-Oki earthquake [Broerse et al., 2011b; Han et al., 2010], which together with the limited amount of data before the earthquake seems inappropriate for analysis. Even in the GRACE data, the detection of the Chile Maule earthquake is a challenge as the earthquake-induced gravity signal is modest compared with hydrology and ice mass loss signals in South America [Han et al., 2010].

[5] The Japan Tohoku-Oki earthquake, which took place on 11 March 2011 at 05:46:23 UTC, was the largest recorded megathrust earthquake in the area of Japan, with the possible exception of the AD 869 Jogan earthquake, and released strain accumulated over several hundreds of years [Ozawa et al., 2012]. Its epicenter was located $38^{\circ} 19^{\circ} 19^{\prime \prime}$ North and $142^{\circ} 22^{\prime} 8^{\prime \prime}$ East. Geodetic measurements such as the GPS onsite network, operated by the Geospatial Information Authority of Japan, measured a site displacement of up to $4.3 \mathrm{~m}$ in horizontal and $66 \mathrm{~cm}$ in vertical direction [Simons et al., 2011]. The coseismic slip area extends approximately $500 \mathrm{~km}$ along the Japan pacific trench [Ozawa et al., 2012]. The detection of such a tremendous event by GOCE gradiometry, which would aim at fine scale gravity structures $(<370 \mathrm{~km}$ spatial scale), could expose unique information for the understanding of local seismic processes in terms of megathrust earthquakes and therefore would be of eminent importance for the region of Japan.

[6] In this paper, we present our analysis of the original GOCE gradient data before and after the Japan Tohoku-Oki earthquake. Han and Ditmar [2008] discuss a spatiospectral localization method for processing geopotential coefficients from satellite gravity mission data where they conclude that events like the 2004 Sumatra-Andaman earthquake are detectable from localized GOCE coefficients. However, no global GOCE gravity field model is available that is purely based on data after the Japan Tohoku-Oki earthquake. We also note that the actual noise level of the GOCE gradients is a factor of 10 greater than assumed in the simulations in Han and Ditmar [2008], [see Ditmar et al., 2003]. We therefore analyze the along-track gravity gradients using tailored outlier detection and optimized filtering in a regional approach.

[7] Analysis of the first release of GOCE data reveals that these suffer from systematic errors at spatial resolutions where the earthquake signals might be visible. The errors are significantly reduced in the recently released reprocessed GOCE gravity gradients [Stummer et al., 2012]. Nevertheless, the expected gravity gradient change is small at satellite altitude compared with the measurement noise level. We therefore analyze spatially averaged and Gaussian smoothed gradient residuals relative to a reference gravity field model. The GOCE gradients are given in the gradiometer reference frame $(X, Y, Z)$ with roughly $X$ along-track, $Y$ cross-track, and $Z$ in radial direction. Because the along-track and cross-track direction are significantly different for ascending and descending tracks, the $V_{X X}$ and $V_{Y Y}$ gradient residuals cannot be simply averaged. The $Z$ direction, in contrast, is almost equal for ascending and descending tracks [Fuchs and Bouman, 2011], and $V_{Z Z}$ can readily be averaged for all tracks. A further reduction in noise is achieved by using the Laplace equation
$V_{Z Z}=-V_{X X}-V_{Y Y}$. This allows averaging the $V_{X X}, V_{Y Y}$, and $V_{Z Z}$ gradients for ascending and descending tracks, resulting in a combined $V_{Z Z}$ which has decreased noise compared with the originally measured $V_{Z Z}$.

[8] The paper is organized as follows. Section 2 discusses the preprocessing of the currently available reprocessed GOCE data. Section 3 discusses the forward modeling of the Japan Tohoku-Oki event. Section 4 evaluates the gravity gradients before and after earthquake occurrence. Section 5 concludes our work.

\section{Data Availability and Data Postprocessing Accuracy}

[9] GOCE data are available from 1 November 2009 onward. We used approximately 12 months of reprocessed (release two) data before and after the earthquake, excluding special events and satellite outages. To identify outliers effectively, we apply a tailored outlier detection, which involves a $3 \sigma$ threshold derived by the median absolute deviation of the residual data distribution with respect to the reference model GOCO03s [Mayer-Gürr et al., 2012]. This global gravity model is used as reference because it incorporates 5 years of SLR observations, 18 months of GOCE gradiometry data, where approximately 1.5 month overlaps with the period after the earthquake (for a discussion see section 4), and 7.5 years of GRACE data, where the processing strategy follows the same approach as for the GOCE time-wise model [Pail et al., 2011]. We believe this procedure has only minor effect on our analysis since the $3 \sigma$ threshold criterion is $>4 \mathrm{mE}$, which is well above the earthquake signal of $0.6 \mathrm{mE}$ or less (see section 3 ), and the detected outliers show no geographical correlation in the region of Japan and its surroundings. The total amount of data is being reduced in comparison with the L1b flagging [Bouman, 2004; Bouman et al., 2009] by about $~ 5 \%$ on average using the additional applied outlier rejection. Furthermore, we use the trace criterion $\left(V_{X X}+V_{Y Y}+V_{Z Z}=0\right)$ to identify outliers.

[10] Four of the six gravity gradients are measured with high accuracy especially in the measurement bandwidth between 5 and $100 \mathrm{mHz}$. The noise level of the accurate $V_{X X}$ and $V_{Y Y}$ gradients is approximately half the noise level of $V_{X Z}$ and $V_{Z Z}$, where $X, Y, Z$ are coordinates in the gradiometer reference frame (GRF). We use a combined version of three of the four accurate GOCE measured gravity gradients in the GRF. As the sum of the three diagonal gradients $V_{X X}, V_{Y Y}$, and $V_{Z Z}$ is to be zero because of the Laplace equation, we can average the three components to obtain a combined $V_{Z Z}$, which significantly reduces the noise compared with the originally measured $V_{Z Z}$ component. Combining the measured gradients $V_{X X}, V_{Y Y}$, and $V_{Z Z}$ results in

$$
V_{Z Z}^{c}=\frac{V_{Z Z}-V_{X X}-V_{Y Y}}{2}
$$

Because the noise level of $V_{Z Z}$ is approximately twice that of $V_{X X}$ and $V_{Y Y}$, it holds that

$$
\sigma:=\sigma_{X X}=\sigma_{Y Y}=\frac{1}{2} \sigma_{Z Z}
$$



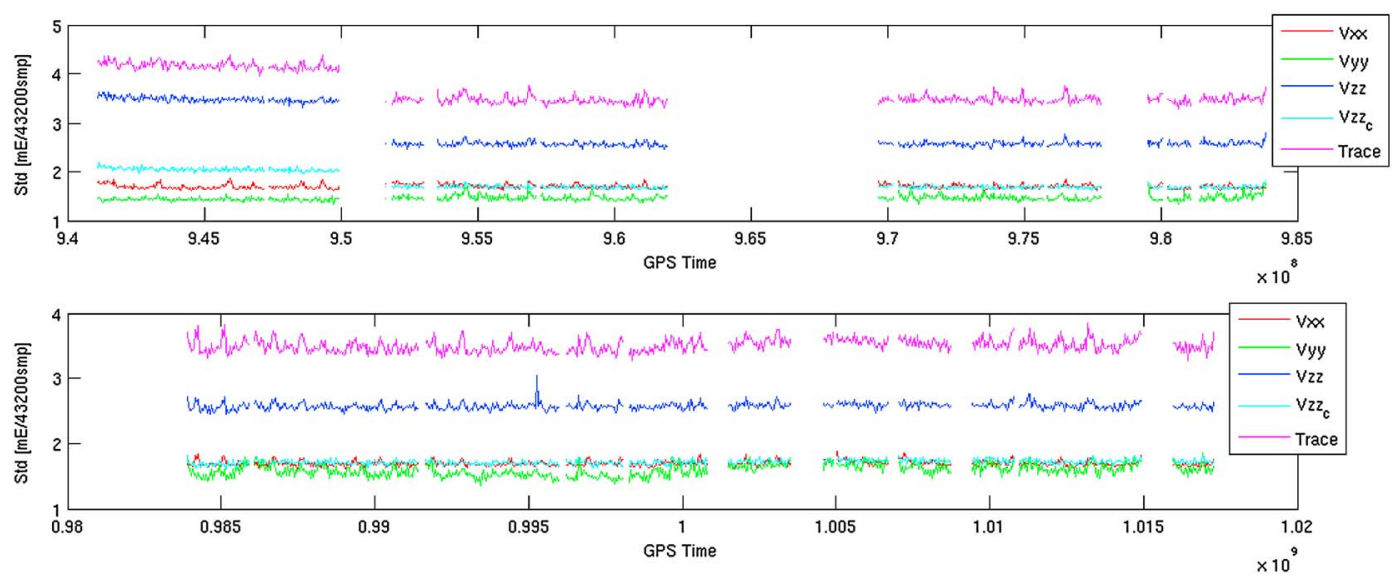

Figure 1. (top and bottom) Residuals of GOCE observations inside the measurement bandwidth of 4.5$30 \mathrm{mHz}$ for the period before and after the earthquake (top and bottom figure respectively). Each data point represents an interval of half a day, which is equal to 43200 samples.

[11] Under the assumption that the errors are uncorrelated, error propagation then gives

$$
\sigma_{Z Z}^{c}{ }^{2}=\frac{1}{4}\left(\sigma_{X X}^{2}+\sigma_{Y Y}^{2}+4 \sigma_{Z Z}^{2}\right)=\frac{6}{4} \sigma^{2}
$$

or

$$
\sigma_{Z Z}^{c}=1.22 \cdot \sigma
$$

[12] Ideally, the combined $V_{Z Z}^{c}$ component therefore results in a noise reduction of the original measured $V_{Z Z}$ component by a factor of 1.64. This is confirmed analyzing the gradient residuals, which are shown in Figure 1. Also, from a statistical distribution (not shown here), we obtain a factor of 1.67 comparing the averaged $V_{X X}, V_{Y Y}$, and $V_{Z Z}$ with the noise level of the original $V_{Z Z}$. This also implicitly justifies the assumption of uncorrelated errors in the diagonal gradients.

[13] The combination of three measurements also circumvents a rotation of the gradiometer reference frame to an Earth-fixed local frame where model information must be incorporated to perform the rotation [Fuchs and Bouman, 2011]. Note that the rotational alignment of the GRF $V_{Z Z}$ component to the radial component (coming from the geocenter) is less than $2.2^{\circ}$ (roll) and $1.0^{\circ}$ (pitch) [Fuchs and Bouman, 2011]. Therefore, the misalignment of the GRF and the radial direction has only minor influence when residual observations (GOCE observations minus GOCO03s) are being used.

[14] For the period before the earthquake, three anomalous events interrupted the nominal satellite operation, which resulted in a data loss of in total 4 months (Figure 1, top). Additionally, for the first anomaly in February 2010, a switch from the onboard CPU-A (Central Processing Unit) to CPU-B took place, which caused a change in the $V_{Z Z}$ gradient quality. The gradient quality for the period after the earthquake is almost constant over time (see Figure 1). The after earthquake period has no major data gaps besides a higher amount of flagged data in November 2011. As a consequence, the total amount of collected data before and after the earthquake is approximately equal (30,497,703 and 32,799,314 samples, respectively).

[15] The spectral gradient error behavior of the release two data shows a much higher consistency and an increased accuracy, especially in the $V_{Y Y}$ component, compared with GOCE release one data. This can be seen from a 90 days spectral density (SD) plot shown in Figure 2 (as a ruleof-thumb, spherical harmonic degree $L$ and frequency $f$ are related as $f \times 5400=L$, where one orbital revolution takes about $5400 \mathrm{~s})$. The gradient residuals, with respect to GOCO03s (blue), show in the lower measurement bandwidth an increased accuracy compared with the quick-look error SD (green) of the release one data. The quick-look gravity field solutions were computed with short latency for GOCE quality assessment [Mayerhofer et al., 2010]. The decreased noise in the lower measurement bandwidth is the result of an improved L1b processing [Stummer et al., 2012] of the second release GOCE data.

[16] Since it is not known (and difficult to evaluate) how the systematic gradiometer errors in the lower measurement bandwidth accumulate, several filter setups have been chosen. First, we use the measurement bandwidth as specified by ESA, $5-100 \mathrm{mHz}$, but use $50 \mathrm{mHz}$ as upper limit. For GOCE, $50 \mathrm{mHz}$ roughly corresponds to spherical harmonic degree and order 270 or a spatial resolution of $74 \mathrm{~km}$. For comparison, we estimated the earthquake-induced $V_{Z Z}$ signal from degree and order 180 to $450(\sim 33-84 \mathrm{mHz})$ at GOCE orbit height using our forward model discussed in section 3. We find that this signal is below $0.005 \mathrm{mE}$, and therefore, it is not expected that an earthquake signal is measurable at satellite altitude above $50 \mathrm{mHz}$. A second parameter set optimizes the global signal-to-noise ratio (SNR) in the bandwidth of $\sim 3.9-30 \mathrm{mHz}$ as derived by Fuchs and Bouman [2011]. Finally, we use a matched filter approach to determine bandwidth limits, which gives a bandwidth of $\sim 4.5-17.5 \mathrm{mHz}$. The matched filter approach is discussed in section 3 and maximizes the expected signal (from the forward computation) considering the spectral behavior of the noise in the observations. Table 1 summarizes the parameters used for the along-track filtering and the spatial smoothing.

[17] The gradient set we use is in summary obtained by subtracting the reference field GOCO03s from the original GOCE observations, outlier detection, and computing the averaged $V_{Z Z}^{c}$ component as discussed above. These gradients are along-track filtered in forward and reverse direction (to preserve phase information) using the three different 

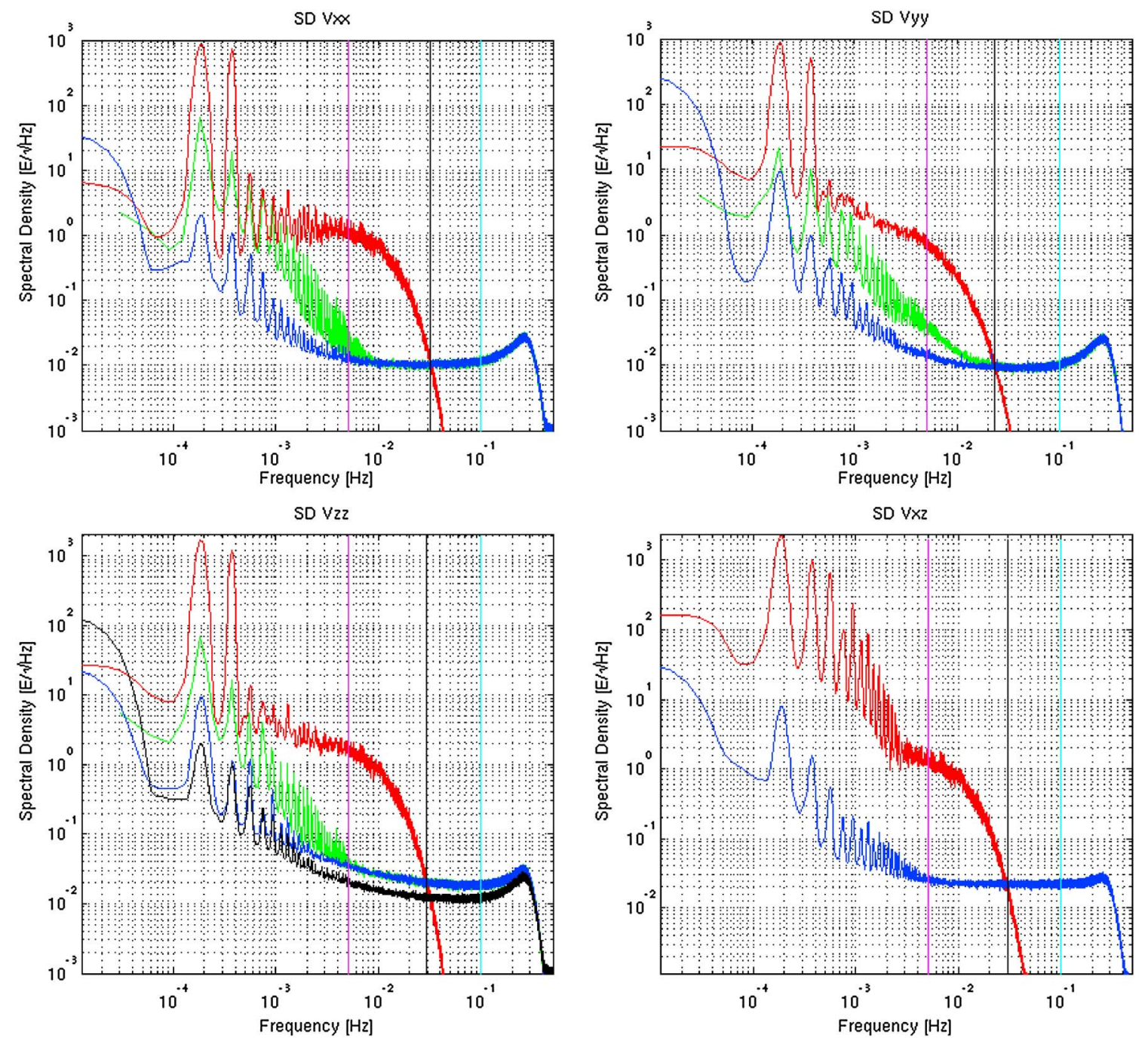

Figure 2. Spectral density of signal and accuracy of the four accurate GOCE gravity gradients derived from 90 days of global data: $V_{X X}, V_{Y Y}, V_{X Z}$, and $V_{Z Z}$ (clockwise). Gravity gradient signal in red, error from quick-look processing in green, residuals to GOOC03S of reprocessed data in blue. The black line (horizontal) shows the residuals to GOOC03S of the combined version of $V_{Z Z}$ (lower left plot). The black line (vertical) marks the crossing frequency where the signal intersects the noise floor $(\mathrm{SNR}=1)$. Magenta and cyan lines indicate the measurement bandwidth $(5-100 \mathrm{mHz})$.

bandwidths defined earlier. The filtered residuals are regionally binned in $0.5^{\circ}$ grid cells where a 2-D Gaussian smoothing has been applied. The filter width of the Gaussian kernel (see Table 1) is chosen for setup 1 in accordance with the smallest along-track frequency where we stick to $111 \mathrm{~km}$ radial distance. For setup 2 and 3, the Gaussian kernel has been chosen with a spatial resolution of $222 \mathrm{~km}$ radial distance.

\section{Forward Modeling}

[18] To forward model the coseismic solid Earth response, we use the publicly available fault slip model of Wei et al. [2011]. Our forward model uses a semi-analytic normal mode model where the Earth is represented as a spherically multilayered and self-gravitating body with a compressible elastic rheology [Sabadini and Vermeersen, 2004]. The elastic stratification of

Table 1. Three Different Along Track and Spatial Filter Parameter Setups

\begin{tabular}{|c|c|c|c|c|c|c|}
\hline & \multicolumn{2}{|c|}{ Setup 1} & \multicolumn{2}{|c|}{ Setup 2} & \multicolumn{2}{|c|}{ Setup 3} \\
\hline & $f_{-}$Low & $f_{-}$High & $f_{-}$Low & $f_{-}$High & $f_{-}$Low & $f_{-}$High \\
\hline$f[\mathrm{mHz}]$ & 5 & 50 & 3.9 & 30 & 4.75 & 17.5 \\
\hline$\sim \mathrm{SH}$ degree and order & 27 & 270 & 21 & 162 & 26 & 95 \\
\hline Wavelength $(\mathrm{km})$ & 742 & 74 & 951 & 124 & 781 & 212 \\
\hline Gaussian kernel sigma (half width) & \multicolumn{2}{|c|}{$111 \mathrm{~km}$} & \multicolumn{2}{|c|}{$222 \mathrm{~km}$} & \multicolumn{2}{|c|}{$222 \mathrm{~km}$} \\
\hline
\end{tabular}



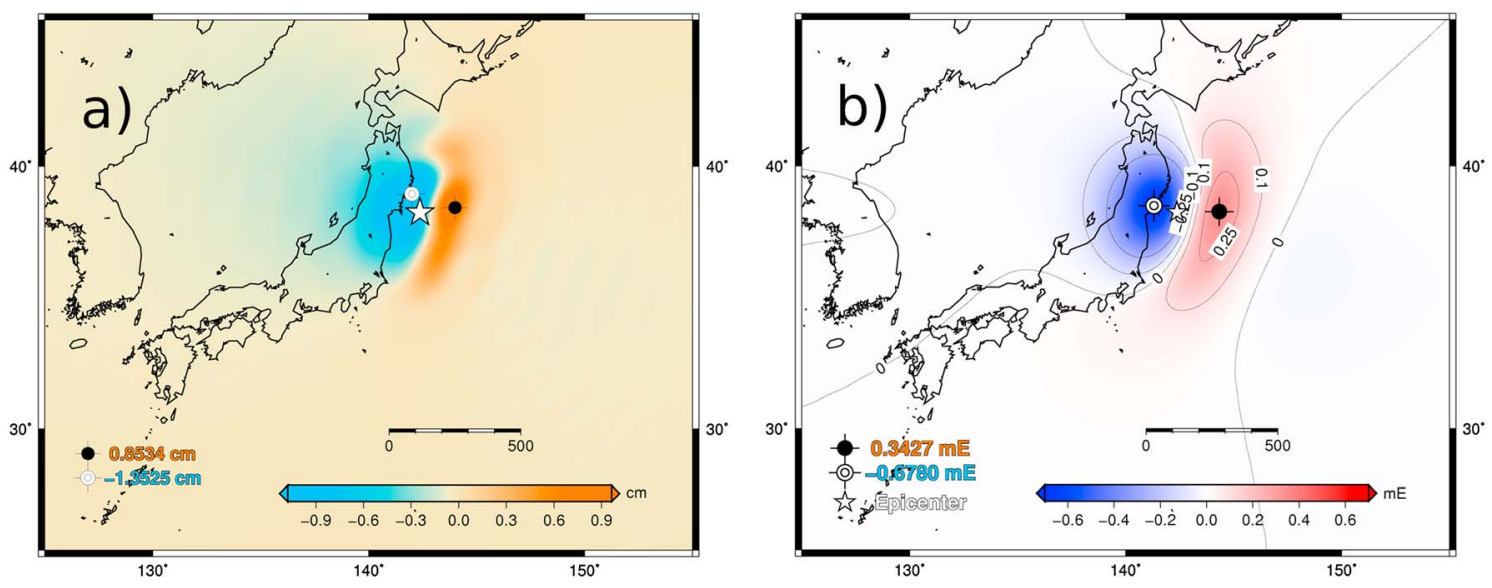

Figure 3. (a) Geoid height change of the forward computation. (b) Gravity gradient signal at $260 \mathrm{~km}$ height derived from the forward computation. Both plots are developed up to spherical harmonic degree and order 250.

the solid earth model is given in Appendix A. The sea-level equation is used to account for the contribution of coseismic ocean water redistribution on gravity changes. A detailed description of the forward modeling can be found in Broerse et al. [2011a, 2011b]. The predicted coseismic gravity change, expressed in geoid heights, results in 0.8 to $-1.2 \mathrm{~cm}$ for the Wei slip model developed to spherical harmonic degree and order 250 which corresponds to $80 \mathrm{~km}$ spatial resolution (see Figure 3 ). The coseismic gravity change is about $\pm 300 \mu \mathrm{Gal}$ [Bouman et al., 2014]. Our modeled results are lower compared with Zhou et al. [2012] who found up to $2.5 \mathrm{~cm}$ geoid change and a coseismic gravity change of $-1000-600 \mu \mathrm{Gal}$. However, Zhou et al. [2012] only show the solid earth contribution. The ocean contribution is of the same order as the solid earth contribution, and taking the former into account gives geoid and gravity anomaly changes that are comparable with our values (Zhou, personal communication, 2012).

[19] The spherical harmonic spectrum (Figure 4) of the obtained geoid heights from forward modeling reveals that up to spherical harmonic degree and order 30 the cumulative geoid change in the vicinity of the epicenter is $1.5 \mathrm{~mm}$, which is only about $10 \%$ of the total RMS (root-mean-square) signal derived up to degree and order 250 . Half of the geoid change signal is located between degree and order 20 to 140 , which roughly equals the along-track spectral region of $4-26 \mathrm{mHz}$ that corresponds approximately with the lower measurement bandwidth in which GOCE is most sensitive. In contrast, monthly GRACE solutions are truncated at spherical harmonic degree and order 60 or 90 and might not be capable to reach even $10 \%$ of the total signal because additional $350 \mathrm{~km}$ Gaussian filtering has to be applied to avoid the prominent GRACE stripes present in the monthly gravity field solutions [e.g., Matsuo and Heki, 2011]. The stripes are clearly visible comparing GRACE and GOCE [Bouman and Fuchs, 2012].

[20] Figure 5 shows the strength and uniqueness of GOCE data. Shown is the expected coseismic gravity change at the Earth's surface developing the model information from degree and order 54 to 180 (which corresponds to GOCE sensitivity) and to degree and order 60 (which corresponds to GRACE sensitivity). We see that, compared with GRACE, the expected coseismic gravity signal maps to the GOCE data with a much higher amplitude and spatial detail.

[21] The obtained geoid series expansions have been used to compute gravity gradient observations along the GOCE orbit for the period 11 March 2011 to 31 March 2012. The gradient signal developed from the forward computed model up to degree and order 450 (see Figure 4) is at GOCE orbit height in the order of $0.6 \mathrm{mE}$ [Bouman et al., 2014]. The forward computed gradients undergo the same processing procedure as explained in section 2.

[22] The GOCE measurements are along-track filtered to reduce the observation noise, which causes the expected gravity gradient signal to decrease dependent on the filter bandwidth. The filtering also redistributes the signal power along the tracks, which are North-South oriented. The forward computed gradients differ especially in amplitude for each setup. For example, while Wei's model predicts for setup 1, a change in $V_{Z Z}$ of $-0.14 \mathrm{mE}$, the same model predicts for setup 3 only $-0.036 \mathrm{mE}$. Also note that the modeled geoid height is an East-West dipole, whereas the along-track filtered gradients from forward modeling have three extremes that are North-South distributed (see Figure 6).

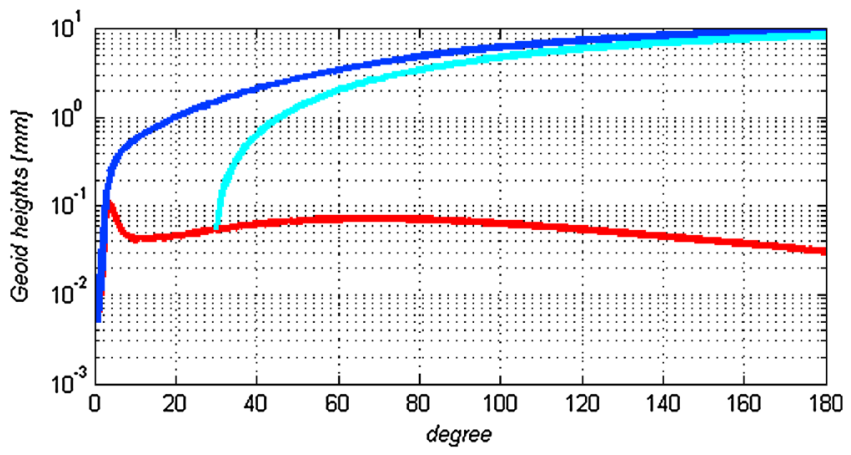

Figure 4. Signal degree geoid spectrum of the forward computation. In red, the RMS signal per spherical harmonic degree is shown. The cumulative RMS geoid signals are shown starting at degree and order 0 (blue) and starting at degree and order 31 (cyan). 

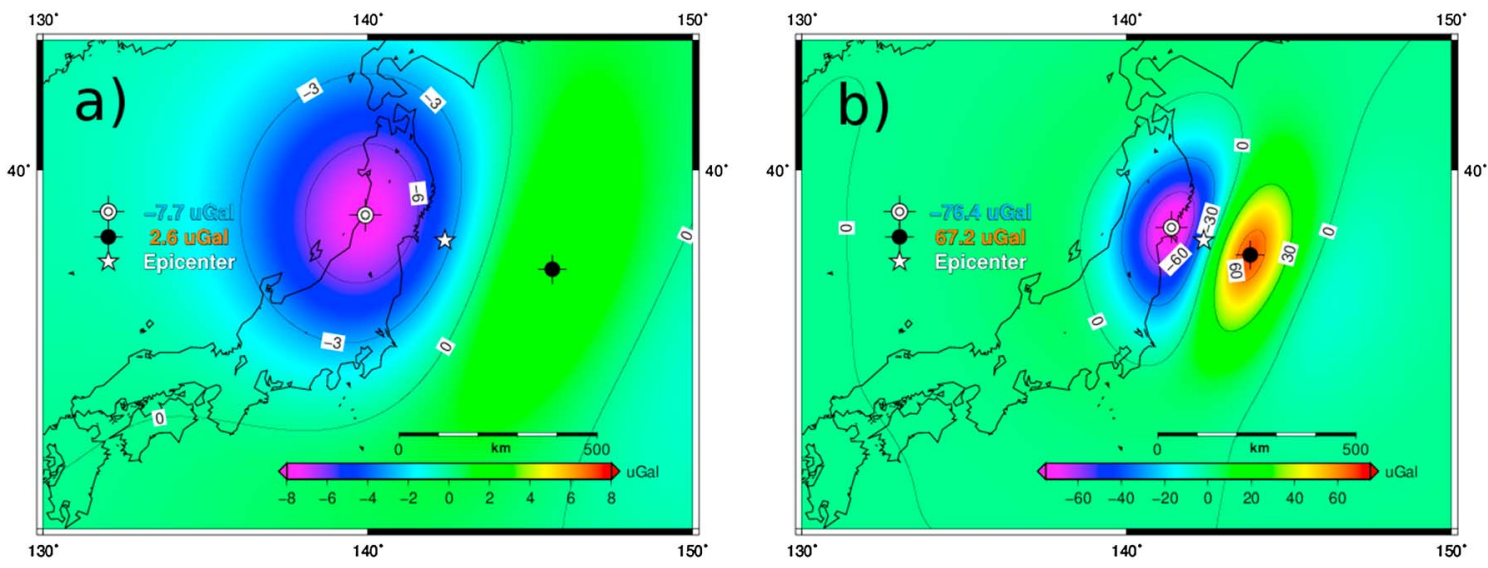

Figure 5. (a) Gravity anomaly signal at the Earth's surface as expected to be measured by GRACE with a Gaussian $300 \mathrm{~km}$ smoothing applied. (b) Gravity signal as expected to be measured from GOCE with a Gaussian band-pass filter 111-300 km applied (right). Note: The color scale of both plots differ by a factor of 9.4 .

[23] The optimal along-track filtering of the GOCE gradients is based on a trade-off between the (expected) spectral signal energy $X(f)$, derived from the modeled gradients, and the spectral gradient noise characteristic $N(f)$, which has been derived from the residuals to the reference field

$$
Y(f)=X(f)+N(f),
$$

with $f$ the frequency. To optimize the signal-to-noise ratio (SNR) of the along-track measured signal $Y(f)$ in the frequency domain, we make use of the Wiener-filter approach

$$
\left|H_{\mathrm{WF}}(f)\right|=\left|\frac{X(f)^{2}}{X(f)^{2}+N(f)^{2}}\right| .
$$

[24] Figure 7 shows in red the signal SD (derived from the forward computation) and in blue the inverse of the noise (error) SD (residuals to the reference field), both normalized to the maximal amplitude of the SD which is in case of $S(f)_{\max }$ $3.6 \times 10^{-4} \mathrm{E} / \mathrm{sqrt}(\mathrm{Hz})$ and for $N(f)_{\max } 2.1 \times 10^{-2} \mathrm{E} / \mathrm{sqrt}(\mathrm{Hz})$. Note that the normalized inverse of the $V_{Z Z}$ error is being shown in Figure 7 because this corresponds to the spectral weight. The corresponding filter frequency response $H_{\mathrm{WF}}$ $(f)$, derived from the spectral signal and noise characteristics, is shown in gray. The approximate frequency limits of the derived Wiener filter are summarized in Table 1.

\section{Evidence of Gravity Change in GOCE Derived Gravity Gradients}

[25] Gravity gradients before and after the Japan TohokuOki earthquake have been analyzed in regional maps of $25^{\circ}$ to $45^{\circ}$ North and $125^{\circ}$ to $155^{\circ}$ East, where in these maps a more specific area of interest (AOI) has been defined as a spherical patch around the epicenter with a radius of $1000 \mathrm{~km}$ ground distance (see dotted circles in Figure 6). The different setups, as explained in sections 2 and 3 and summarized in Table 1, represent thereby different resolutions and accuracies derived from the gridded data.

[26] The gridded samples before earthquake occurrence cover the time span of 1 November 2009 till 11 March 2011.
Because the reference model GOCO03s includes 18 months of GOCE data from November 2009 till April 2011, it is assumed that the gradient observations for the period before the earthquake (1 November 2009-11 March 2011) are modeled best by this global spherical harmonic representation, and it is to be expected that the gradient residuals before earthquake occurrence do not significantly differ from zero because the earthquake-induced signal for a $\sim 1.5$ month period is below the signal-to-noise ratio of the 18 months average. This is confirmed analyzing regional maps (see Figures 8a, $8 \mathrm{c}$, and 8e), although the GOCE data in GOCO03s stem from release one and we use reprocessed data here.

[27] Regionally, we tested the null hypothesis that the $V_{Z Z}^{c}$ residuals of the along-track data for setup 1 are Gaussian distributed. This is confirmed using a chi-square fit where a confidence level of $95 \%$ was chosen. The standard deviations of the GOCE measurements before and after earthquake occurrence have been computed in two different ways (1) from the residuals to the reference model and (2) from data accumulated inside the $0.5^{\circ}$ grid cells. Because the two values only marginally differ $(<0.1 \mathrm{mE})$ for all setups, only the former are shown in Table 3.

[28] The regional analysis of Japan reveals that the local gradient accuracy is homogeneous. Therefore, the accuracy derived from the along-track data (shown in Table 3) is restricted to the AOI as shown in the local maps of Figure 6. Globally, in contrast, the gradient accuracy is heterogeneous because close to the magnetic poles (Greenland and south of Australia) the $V_{Y Y}$ component shows anomalous behavior [Bouman and Fuchs, 2012], which is lower for the reprocessed data but still present.

[29] On average, there are between 114 and 122 samples in a half degree grid cell for the period before the earthquake and between 112 and 114 samples for the period after the earthquake. As we apply a bandwidth specific flagging, these numbers are not equal for the three setups. Because of the degraded $V_{Z Z}$ accuracy between November 2009 and January 2010, a larger amount of samples before the earthquake is needed to obtain almost the same averaged accuracy as after the earthquake. The deviation of the mean in $0.5^{\circ}$ grid cells after Gaussian weighting is given in the last two columns of 


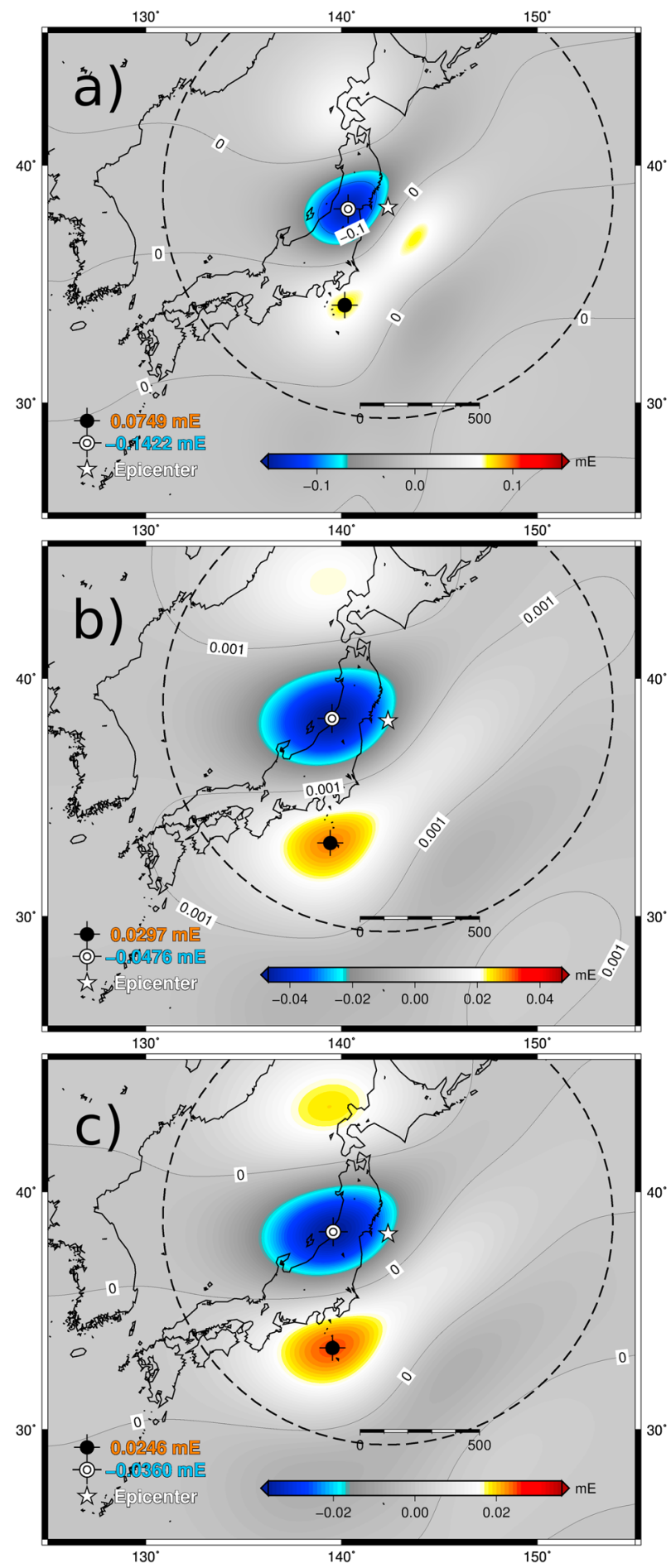

Figure 6. Computed $V_{Z Z}$ signal for 11 March 2011-31 March 2012 at GOCE altitude using the Wei slip model. The same processing as for the GOCE observations has been applied. The dashed black lines indicate the area of interest. (a) Setup 1, (b) setup 2, and (c) setup 3 are displayed. Color bars have been scaled to the maximum amplitude.

Table 2. The $1 \sigma$ values are $0.052 \mathrm{mE}$ (setup 1), $0.021 \mathrm{mE}$ (setup 2), and $0.015 \mathrm{mE}$ (setup 3) for the data before the earthquake. Given the extremes $-0.142 \mathrm{mE},-0.052 \mathrm{mE}$, and $-0.036 \mathrm{mE}$ of the modeled gradients for setup 1, 2, and 3 respectively, the expected detection capability is in the order of $2.7 \sigma(\operatorname{setup} 1), 2.5 \sigma(\operatorname{setup} 2)$, and $2.5 \sigma(\operatorname{setup} 3)$. The corresponding confidence levels are $99 \%, 98.8 \%$, and $98.8 \%$. It should be noted, however, that our analysis of the gradient residuals, discussed below, indicates that systematic errors may be present in the gridded data.

[30] We analyzed regional maps of the residuals before and after earthquake occurrence for the three different spatial setups, see Figure 8. Setup 1 has the highest spatial resolution up to $110 \mathrm{~km}$. For the period before the earthquake, we would expect in case of stationary noise a homogenous error pattern. We see, however, mainly over the ocean significant small scale deviations that are greater than $3 \sigma$ indicated by the red and blue colors (Figures $8 \mathrm{a}, 8 \mathrm{c}$, and 8e). These could be errors caused, e.g., by the higher noise level in the first 3 months in combination with insufficient Gaussian smoothing, or residual signals because of inadequate background models. For the period after earthquake occurrence, a gradient pattern above $3 \sigma$ is visible in the residual map, which resembles the modeled signal in the top panel of Figure 6. For setup 2 and 3 , the spatial resolution is lower than for setup 1 because of the $2^{\circ}$ Gaussian smoothing used in combination with a smaller cutoff frequency. This is probably the reason that the residuals before the earthquake are almost always below $3 \sigma$. The larger residuals in the South might be related to the lower track density toward the equator. In the residual maps, for the period after earthquake occurrence, North-South distributed statistically significant extremes are visible for setup 2 and 3 .

[31] Using the defined AOI (1000 km radial ground distance to epicenter), we computed the Pearson correlation coefficient for the model and the measurements. This analysis reveals the model fit to the observations, which is a measure of the particular local match. All three setups have been compared (see Table 3), where correlation coefficients above 0.5 have been obtained. A maximum correlation coefficient of $\sim 0.7$ has been found for setup 2, which indicates that the modeled and measured signals are significantly spatially correlated.

[32] All residuals become smaller subtracting the modeled gradients (Figure 6) from the observed gravity gradients (Figure 8). Additional tests show that the residuals can be

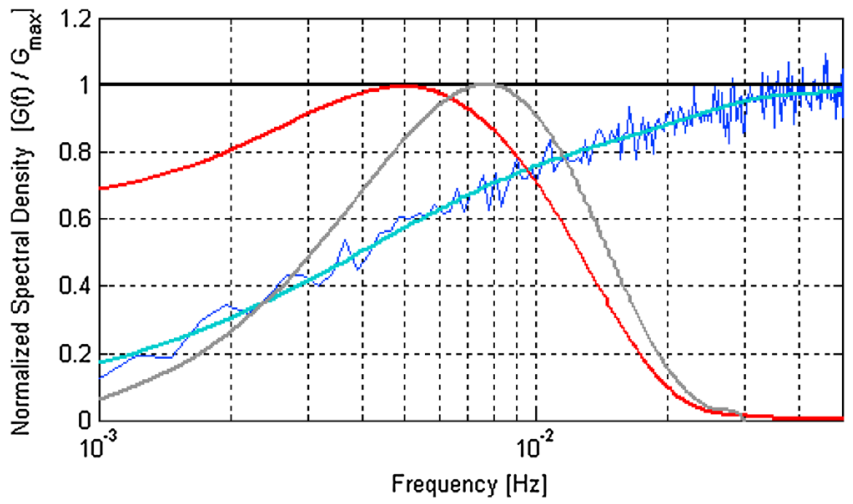

Figure 7. Inverse normalized spectral density of $V_{Z Z}$ errors in blue and normalized spectral density expected $V_{Z Z}$ coseismic earthquake signal in red from forward computation along the GOCE orbit. The cyan curve is obtained by a ninth order polynomial interpolation of the $V_{Z Z}$ errors. All spectral densities have been normalized with the absolute value of the maximum signal amplitude. The Wiener filter frequency response is displayed in gray. The black horizontal bar indicates one. 


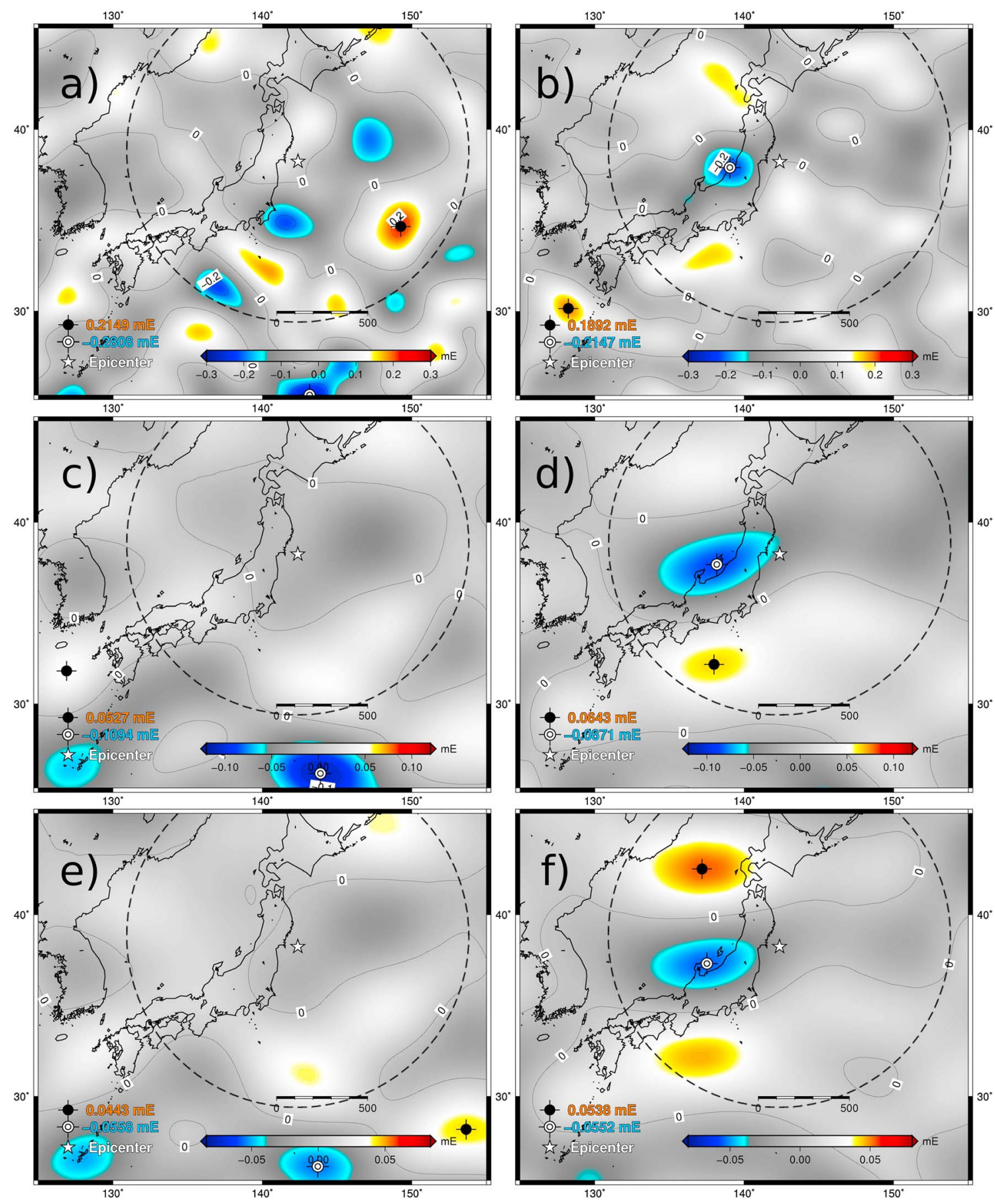

Figure 8. (a and b) Binned residuals for setup 1, (c and d) setup 2, and (e and f) setup 3 for the period before the earthquake (left column) and after the earthquake (right column). All values below a $3 \sigma$ threshold are depicted with gray colors, where $\sigma$ is the deviation of the mean given in the last two columns of Table 2. The star indicates the location of the earthquake epicenter.

further reduced assuming a 1.3 times higher gradient signal as proposed by our forward computation. The amplitudes in the observed residuals are therefore greater than the modeled coseismic signals. This may be related to the postseismic changes that are in the same sense as the coseismic changes for a few months as analysis of the GRACE data showed [Heki et al., 2012]. The problem, however, is not straightforward. Long-term postseismic changes may have a different polarity and start to cancel the coseismic changes. In addition, the geographical distribution of gravity changes in the coseismic, short-term postseismic, and long-term postseismic stage are somewhat different from each other [Heki et al., 2012]. The differences between our forward model and the GOCE measurements may also be caused by observational noise or simplifications in the forward model (see the discussion in the next section), but are not well understood.

[33] We also observe that the extremes from GOCE in Figure 8 are shifted toward the South-West by roughly $100 \mathrm{~km}$ 
Table 2. Gradient Properties Derived Inside the AOI for the Setups Before and After Earthquake Occurrence ${ }^{\mathrm{a}}$

\begin{tabular}{|c|c|c|c|c|c|c|}
\hline \multirow[b]{2}{*}{$V_{Z Z}^{c}$ Properties Inside AOI } & \multicolumn{2}{|c|}{ Std. Along-Track Residuals (mE) } & \multicolumn{2}{|c|}{ Avg. Samples Per Half Degree Grid Cell } & \multicolumn{2}{|c|}{ Deviation of the Mean $(1 \sigma)(\mathrm{mE})$} \\
\hline & Before & After & Before & After & Before & After \\
\hline Setup 1 & 2.32 & 2.20 & 112 & 117 & 0.052 & 0.049 \\
\hline Setup 2 & 1.82 & 1.71 & 113 & 118 & 0.021 & 0.019 \\
\hline Setup 3 & 1.33 & 1.22 & 114 & 120 & 0.015 & 0.013 \\
\hline
\end{tabular}

${ }^{\mathrm{a}}$ The deviation of the mean represents the $1 \sigma$ criterion after smoothing.

compared with their location in Figure 6, which is based on modeling. A similar observation is made when comparing a GPS-based slip model with a slip model that is based on a joint inversion of GRACE and GPS data [Feng et al., 2013].

\section{Conclusions and Discussion}

[34] We assessed the possibility of detecting the coseismic gravity change for the Japan Tohoku-Oki earthquake by GOCE gravity gradiometry. The fault slip model of $\mathrm{Wei}$ et al. [2011] has been used to predict vertical gravity gradients along a nominal GOCE orbit. The forward computation shows that the coseismic gravity signal is above the observational accuracy of GOCE gravity gradiometry.

[35] For the comparison of the modeled gravity gradients with the GOCE observations, we used a combination of three of the four accurate measured GOCE gravity gradients $V_{X X}$, $V_{Y Y}$, and $V_{Z Z}$ where an error reduction of a factor of 1.67 is being achieved compared with the original measured $V_{Z Z}$ component. In addition, we use three different along-track and spatial averaging schemes. The comparison at gradient level of the signal before and after the earthquake reveals that there is a statistical significant signal in the GOCE combined $V_{Z Z}$ gravity gradient at a confidence level of at least $95 \%$. In a correlation study of gravity gradient signal to the forward modeled signals, a maximum correlation factor of $\sim 0.7$ was found, which also indicates the presence of earthquakeinduced gravity signal in the GOCE gradients for a predefined area of interest.

[36] Discrepancies between the observations and the forward modeled signal are present and are currently under investigation. They may be caused by systematic errors in the GOCE data, and also by errors in the background models such as the ocean model for circulation and tides that has been used to account for oceanic mass variations. The latter are strong in the vicinity of Japan because of the western boundary Kuroshio Current.

[37] Hydrology signals may influence the observed gravity gradients, but a test using Global Land Data Assimilation System [Rodell et al., 2004] shows only small signals around Japan, at maximum 5\% of the negative peak of the gravity gradients in the unfiltered forward model, and negligible over the ocean. Finally, our forward modeling may contain errors, although a comparison for monthly GRACE solutions revealed no significant amplitude differences.

[38] In this paper, we based our forward analysis on one of the available slip solutions; however, in recent literature, there is evidence that the fault ruptured all the way to the trench [e.g., Hooper et al., 2012]. These claims are based on seafloor displacements and tsunami modeling, which is not accounted for in the Wei et al. slip model. Downdip afterslip and possible large amounts of shallow coseismic slip, which is not included in the forward model, may partly explain the current mismatch with the observed GOCE gravity gradients.

[39] Because of averaging 13 months of data after earthquake occurrence, any large scale solid Earth deformation as a reaction to the main event ends up in the averaged gravity gradients, by mechanisms such as frictional afterslip (continued aseismic slip on the rupture or adjacent fault segments) or viscoelastic relaxation of the upper mantle (viscoelastic creep below the elastic lithosphere). The relative contribution of afterslip and viscoelastic mantle relaxation and the time evolution of these two processes are still widely under debate for megathrust earthquakes [Panet et al., 2010; Hoechner et al., 2011; Shearer and Bürgmann, 2010].

[40] Ozawa et al. [2012] report postseismic deformation (as observed by GPS) that peaks in the first two months after the earthquake and gradually decays in the 7 months taken into account. The authors interpret the postseismic deformation as being caused by afterslip, which extends the total slip area downdip and resembles an estimated seismic moment of $18 \%$ of the main shock. As the postseismic deformation peaks in the first months following the earthquake, its effect on gravity is almost completely absorbed in our gravity gradient averages. As averaging several months of GOCE data is needed, it is therefore not possible to separate postseismic from coseismic gravity gradient changes.

[41] This study indicates for the first time that it is possible to detect coseismic gravity changes by GOCE spaceborne gradiometry, originated from the Japan Tohoku-Oki earthquake. GOCE provides as independent data source valuable information on the total mass displacement for such a tremendous event. Especially for this occasion, a large part of the signal is being located at small scales, where the GRACE mission might need longer integration periods or simply is not sensitive. We expect that through the higher sensitivity at small scales, new applications in seismic constraints using GOCE data could be developed. A combination of GRACE and GOCE information is essential to exploit the best possible mapping of the coseismic gravity change of the Japan Tohoku-Oki earthquake. Furthermore, an extension of the analysis at gradient level to gravity field recovery would allow combining all accurate gravity gradient components in an optimal way, possibly reducing systematic errors and increasing signal-to-noise ratio.

Table 3. Correlation Coefficient Derived From 1307 Grid Cells Correlated Between Observations and Forward Modeled Gradients Inside the AOI

\begin{tabular}{lccc}
\hline & \multicolumn{3}{c}{ Correlation Coefficient Inside AOI } \\
\cline { 2 - 4 } & Setup 1 & Setup 2 & Setup 3 \\
\hline Model versus measurements & 0.53 & 0.73 & 0.64 \\
\hline
\end{tabular}


Table A1. Parameters for the Elastic Earth Model

\begin{tabular}{lcccc}
\hline Layer & $r(\mathrm{~km})$ & $\rho\left(\mathrm{kg} / \mathrm{m}^{3}\right)$ & $\mu\left(\mathrm{N} / \mathrm{m}^{2}\right)$ & $\lambda\left(\mathrm{N} / \mathrm{m}^{2}\right)$ \\
\hline 1 & 6369 & 2732.00 & $0.341 \times 10^{11}$ & $0.395 \times 10^{11}$ \\
2 & 6347 & 3380.00 & $0.677 \times 10^{11}$ & $0.858 \times 10^{11}$ \\
3 & 6311 & 3377.00 & $0.673 \times 10^{11}$ & $0.853 \times 10^{11}$ \\
4 & 6291 & 3375.00 & $0.646 \times 10^{11}$ & $0.862 \times 10^{11}$ \\
5 & 6256 & 3367.00 & $0.637 \times 10^{11}$ & $0.852 \times 10^{11}$ \\
6 & 6151 & 3476.00 & $0.747 \times 10^{11}$ & $1.16 \times 10^{11}$ \\
7 & 5971 & 3858.00 & $1.04 \times 10^{11}$ & $1.63 \times 10^{11}$ \\
8 & 5701 & 4501.00 & $1.75 \times 10^{11}$ & $2.28 \times 10^{11}$ \\
9 & 5200 & 4785.00 & $2.07 \times 10^{11}$ & $2.89 \times 10^{11}$ \\
10 & 4700 & 5050.00 & $2.36 \times 10^{11}$ & $3.50 \times 10^{11}$ \\
11 & 4200 & 5319.00 & $2.66 \times 10^{11}$ & $4.18 \times 10^{11}$ \\
12 & 3630 & 5510.00 & $2.88 \times 10^{11}$ & $4.60 \times 10^{11}$ \\
13 & 3480 & 10931.00 & 0 & $9.42 \times 10^{11}$ \\
\hline
\end{tabular}

\section{Appendix A: Earth Model Stratification}

[42] The parameters we used for the elastic Earth model are given in the table below, where the volume is averaged from preliminary reference Earth model [Dziewonski and Anderson, 1981]. Here $r$ is the distance with respect to the center of the Earth, $\rho$ is the density of the layer, $\mu$ is the rigidity, and $\lambda$ the first Lamé parameter. Between 6371 and $6369 \mathrm{~km}$, an ocean layer is modeled separately. (Table A1.).

[43] Acknowledgment. This work has been done in the framework of the ESA sponsored GOCE + Time-Variations study as part of ESA's Support to Science Element (STSE). Furthermore, Taco Broerse acknowledges financial support from ISES and NWO. We thank S. Wei for providing his fault slip model. The constructive reviews by Shin-Chan Han and Kosuke Heki helped to improve the paper.

\section{References}

Abrikosov, O., F. Jarecki, J. Müller, S. Petrovic, and P. Schwintzer (2006), The impact of temporal gravity variations on GOCE gravity field recovery, in Observation of the Earth System From Space, edited by J. Flury et al., pp. 255-269, Springer, Heidelberg.

Bouman, J. (2004), Quick-look outlier detection for GOCE gravity gradients, Newton's Bull., 2, 77-86.

Bouman, J., and M. Fuchs (2012), GOCE gravity gradients versus global gravity field models, Geoph. J. Int., 189, 846-850, doi:10.1111/j.1365246X.2012.05428.x.

Bouman, J., S. Rispens, T. Gruber, R. Koop, E. Schrama, P. Visser, C. C. Tscherning, and M. Veicherts (2009), Preprocessing of gravity gradients at the GOCE high-level processing facility, J. Geod., 83, 659-678.

Bouman, J., S. Fiorot, M. Fuchs, T. Gruber, E. Schrama, C. C. Tscherning, M. Veicherts, and P. Visser (2011), GOCE gravity gradients along the orbit, J. Geod., 85, 791-805, doi:10.1007/s00190-011-0464-0.

Bouman, J., M. Fuchs, T. Broerse, B. Vermeersen, P. Visser, E. Schrama, and M. Schmidt (2014), Modelling and observing the Mw 8.8 Chile 2010 and Mw 9.0 Japan 2011 Earthquakes using GOCE, in Earth on the Edge: Science for a Sustainable Planet, pp. 173-180, edited by C. Rizos, and P. Willis, IAG Symp. 139, Springer Verlag, Berlin, Heidelberg.

Broerse, D., L. Vermeersen, R. Riva, and W. van der Wal (2011a), Ocean contribution to co-seismic crustal deformation and geoid anomalies: Application to the 2004 December 26 Sumatra-Andaman earthquake, Earth Planet. Sci. Lett., 305, 341-349, doi:10.1016/j.epsl.2011.03.011.

Broerse, D., P. Visser, J. Bouman, M. Fuchs, B. Vermeersen, and M. Schmidt (2011b), Modelling and observing the 8.8 Chile and 9.0 Japan earthquakes using GOCE, Proceedings of the 4th International GOCE User Workshop, ESA Publication SP-696.

Ditmar, P., J. Kusche, and R. Klees (2003), Computation of spherical harmonic coefficients from gravity gradiometry data to be acquired by the GOCE satellite: Regularization issues, J. Geod., 77, 465-477.

Dziewonski, A., and D. Anderson (1981), Preliminary reference Earth model, Phys. Earth Planet. Inter., 25(4), 297-356.

European Space Agency (1999), Gravity field and steady-state ocean circulation mission. Reports for mission selection; the four candidate earth explorer core missions, ESA SP-1233(1).
Feng, W., Q. Li, Z. Li, and T. Hoey (2013), Slip distribution of 2011 Japan Mw 9.0 earthquake determined by GPS-coseismic displacement and GRACE gravity changes, presented at the EGU Scientific Assembly, Vienna, Austria. Fuchs, M., and J. Bouman (2011), Rotation of GOCE gravity gradients to local frames, Geophys. J. Int., 187, 743-753, doi:10.1111/j.1365246X.2011.05162.x.

Fuchs, M., J. Bouman, T. Broerse, B. Vermeersen, and P. Visser (2012), Assessment of the possibility to observe gravity changes due to the Japan-Tohoku 2011 earthquake by GOCE gravity gradiometry, EGU 2012, Wien, Austria, 2012-04-26.

Han, S.-C., and P. Ditmar (2008), Localized spectral analysis of global satellite gravity fields for recovering time-variable mass redistributions, J. Geod., 82, 423-430, doi:10.1007/s00190-007-0194-.

Han, S.-C., C. K. Shum, P. Ditmar, P. Visser, C. van Beelen, and E. J. O. Schrama (2006), Effect of high-frequency mass variations on GOCE recovery of the Earth's gravity field, J. Geodyn., 41, 69-76, doi:10.1016/j.jog.2005.08.005.

Han, S.-C., J. Sauber, and S. Luthcke (2010), Regional gravity decrease after the 2010 Maule (Chile) earthquake indicates large-scale mass redistribution, Geophys. Res. Lett., 37, L23307, doi:10.1029/2010GL045449.

Han, S. C., J. Sauber, and R. Riva (2011), Contribution of satellite gravimetry to understanding seismic source processes of the 2011 Tohoku-Oki earthquake, Geophys. Res. Lett., 38, L24312, doi:10.1029/2011GL049975.

Heki, K., and K. Matsuo (2010), Coseismic gravity changes of the 2010 earthquake in central Chile from satellite gravimetry, Geophys. Res. Lett., 37, L24306, doi:10.1029/2010GL045335.

Heki, K., Y. Mitsui, K. Matsuo, and Y. Tanaka (2012), Accelerated subduction of the Pacific Plate after mega-thrust earthquakes: Evidence from GPS and GRACE, Presented at the AGU Fall Meeting 2012, San Francisco.

Hoechner, A., S. Sobolev, I. Einarsson, and R. Wang (2011), Investigation on afterslip and steady state and transient rheology based on postseismic deformation and geoid change caused by the Sumatra 2004 earthquake, Geochem. Geophys. Geosyst., 12, Q07010, doi:10.1029/2010GC003450.

Hooper, A., et al. (2012), Importance of horizontal seafloor motion on tsunami height for the $\mathrm{Mw}=9.0$ Tohoku-Oki Earthquake, Earth Planet. Sci. Lett., 361, 469-479, doi:10.1016/j.epsl.2012.11.013.

Matsuo, K., and K. Heki (2011), Coseismic gravity changes of the 2011 Tohoku-Oki earthquake from satellite gravimetry, Geophys. Res. Lett., 38, L00G12, doi:10.1029/2011GL049018.

Mayer-Gürr, T., et al. (2012), The new combined satellite only model GOCO03s, Poster presented at the GGHS2012, Venice.

Mayerhofer, R., R. Pail, and T. Fecher (2010), Quick-look gravity field solution as part of the GOCE quality assessment, in Proc. ESA Living Planet Symposium, ESA SP-686.

Ozawa, S., T. Nishimura, H. Munekane, H. Suito, T. Kobayashi, M. Tobita, and T. Imakiire (2012), Preceding, coseismic, and postseismic slips of the 2011 Tohoku earthquake, Japan, J. Geophys. Res., 117, B07404, doi:10.1029/ $2011 \mathrm{JB} 009120$.

Pail, R., et al. (2011), First GOCE gravity field models derived by three different approaches, J. Geod., 85, 819-843, doi:10.1007/s00190-011-0467-x.

Panet, I., F. Pollitz, V. Mikhailov, M. Diament, P. Banerjee, and K. Grijalva (2010), Upper mantle rheology from GRACE and GPS postseismic deformation after the 2004 Sumatra-Andaman earthquake, Geochem. Geophys. Geosyst., 11, Q06008, doi:10.1029/2009GC002905.

Rodell, M., et al. (2004), The global land data assimilation system, Bull. Am. Meteorol. Soc., 85(3), 381-394.

Sabadini, R., and B. Vermeersen (2004), Global Dynamics of the Earth: Applications of Normal Mode Relaxation Theory to Solid-Earth Geophysics, Kluwer Acad. Publ, Dordrecht, The Netherlands.

Shearer, P., and R. Bürgmann (2010), Lessons learned from the 2004 SumatraAndaman megathrust rupture, Annu. Rev. Earth Planet. Sci., 38, 103-131. Simons, M., et al. (2011), The 2011 magnitude 9.0 Tohoku-Oki Earthquake: Mosaicking the megathrust from seconds to centuries, Science, 332, 1421-1425, doi:10.1126/science.1206731.

Stummer, C., C. Siemes, R. Pail, B. Frommknecht, and R. Floberghagen (2012), Upgrade of the GOCE level $1 \mathrm{~b}$ gradiometer processor, Adv Space Res., 49(4), 739-752, doi:10.1016/j.asr.2011.11.027, Elsevier, ISSN 0273-1177.

Wang, L., C. K. Shum, F. J. Simons, B. Tapley, and C. Dai (2012), Coseismic and postseismic deformation of the 2011 Tohoku-Oki earthquake constrained by GRACE gravimetry, Geophys. Res. Lett., 39, L07301, doi:10.1029/2012GL051104.

Wei, S., A. Sladen, and the Aria Group (2011), Updated result: 3/11/2011 (Mw 9.0), Tohoku-Oki, Japan. http://www.tectonics.caltech.edu/slip_history/ 2011_taiheiyo-oki/.

Zhou, X. X., W. Sun, B. Zhao, G. Fu, J. Dong, and Z. Nie (2012), Geodetic observations detecting coseismic displacements and gravity changes caused by the $\mathrm{Mw}=9.0$ Tohoku-Oki earthquake, J. Geophys. Res., 117, B05408, doi:10.1029/2011JB008849. 